\title{
SUPER-RESOLUTION RECONSTRUCTION OF CROP DISEASE IMAGES BASED ON DEPTH LEARNING
}

\author{
WANG, $X$. \\ College of Teacher Education, Pingdingshan University, Pingdingshan 467000, China \\ (e-mail: aaabbbw211@126.com) \\ (Received $3^{\text {rd }}$ Jun 2019; accepted $11^{\text {th }}$ Oct 2019)
}

\begin{abstract}
Current image reconstruction has some problems, such as low image segmentation and denoising precision, slow convergence speed, and poor image integrity after reconstruction. In this regard, this study proposed a super-resolution reconstruction of crop disease images based on deep learning. The improved neighborhood averaging method is used to denoise the low frequency subband image, and the enhanced wavelet coefficients are replaced by the wavelet inverse transform to realize the high frequency subband image denoising. The image enhancement results are introduced, and the image initial segmentation area is obtained by using the color roughness concept and the incremental region growth method. When the stop condition is satisfied, the best result is presented. The small feature extraction of image segmentation results is carried out, and the feature vectors are transformed from low resolution space to high resolution space by nonlinear mapping. Feature extraction, nonlinear mapping and image reconstruction are fused into a deep convolution neural network, and the final reconstruction results are obtained. The experiment showed that the method improves the image segmentation and denoising precision, and that the integrity coefficient of the image reconstruction is high and the reliability is strong.

Keywords: reconstruction, wavelet inverse transform, image denoising, nonlinear mapping
\end{abstract}

\section{Introduction}

The determination and control of crop diseases and insect pests play an important role in the development of agriculture in China. In actual production, most farmers only rely on experience and their own senses to diagnose crop diseases. Although it has achieved some results, it also has harmed the growth status of crops (Burgess et al., 2017). Therefore, early diagnosis is the key to accurately identify disease. It is not scientific to achieve the accurate recognition of all kinds of diseases only by human visual observation and experience (Gao et al., 2017; Fu and Liu, 2017). The symptoms of common crop diseases are mainly expressed by the color and texture of leaves (Mo et al., 2018; Kniat, 2017; Sanchez et al., 2017). At present, the treatment of agricultural diseases is mainly through expert diagnosis, and then taking corresponding measures (Wei, 2017). The symptoms of some diseases are not obvious at the initial stage, their symptoms become more pronounced with time, enabling precise diagnosis. This delays prevention and the time treatment can begin. The symptoms of some diseases are similar to those of other diseases. At this time, the experts have difficulties confirming the diagnosis, and other auxiliary methods should be used. Based on the research situation of crop diseases and insect pests, the following outstanding research results have appeared (Roy et al., 2018; Nongqwenga and Modi, 2017).

Mao et al. reconstruct the SEM (Sitemap) image by using the image edge gradient information. It used Kirsch operator to analyze the gradient information of the image, and then classified each region by using the feature that the gradient of the outer edge of the image is greater than the gradient of the inner edge, and filled the final image according to the statistical information. Experimental results showed that this method has high stability and high automation in high-resolution images, but the accuracy of 
image segmentation is not enough, resulting in image reconstruction effect is not very ideal (Mao and Shi, 2016). Song proposed the 3D (3 Dimensions) reconstruction of images based on compressed sensing. By establishing the mathematical model of the image reconstruction of the SRM section, the principle of the algebraic reconstruction is analyzed. Based on the analysis of ART (Algebra Reconstruction Technique), SART (Simultaneous Algebraic Reconstruction Technique) and SIRT (simultaneous iterative reconstruction technique) method and its iterative formula, the CSART (compressive sensing Algebra Reconstruction Technique) algorithm combining the compressed sensing theory with ART is put forward to test the SRM (Switched Reluctance Moto) structure simulation model. According to the obtained projection data, several algorithms are analyzed and compared. The results showed that the CSART algorithm has the fastest convergence rate, but the reconstructed image integrity is not guaranteed (Song, 2016). Yu proposed a Tabu search based image reconstruction of capacitance tomography. The Tabu algorithm is referenced to the ECT (Emission Computed Tomography) image reconstruction. By constructing the target function, the neighborhood and Tabu table are set to constrain the image reconstruction. The image reconstruction problem is converted to the global optimal solution based on the contempt criteria and the ad hoc criteria and used termination criteria to jump out of Tabu Search. The simulation results showed that the reconstructed image is more fidelity, but the speed of reconstruction is slower (Yu et al., 2016). Li proposed the super-resolution image reconstruction based on support vector machine. The sparse characteristic of the image block is calculated, and the sparse representation coefficient is obtained. Then the sparse representation coefficient is used to train the support vector machine, and the super-resolution image reconstruction model is established. Finally, the simulation is used to analyze its performance. The results showed that the method can effectively maintain the speed of reconstruction, but the noise in the image is larger ( $\mathrm{Li}, 2016)$. Guo proposed the super-resolution image reconstruction based on wavelet denoising and neural network. The wavelet transform is used to remove the noise in the image, improve the image quality, and obtain the learning samples needed for the network training. The learning samples are trained by neural network, and the artificial fish swarm algorithm is used to determine the key parameters of the neural network. Finally, the effectiveness of the super-resolution image reconstruction algorithm is tested, and its superiority is analyzed by comparison experiment. The experimental results showed that the proposed method can achieve an ideal image denoising effect, but there is also a low segmentation precision (Guo and Cen, 2016).

At present, there are various problems in image reconstruction methods of crop diseases, so we proposed to use deep learning to reconstruct super-resolution images of crop diseases. It can solve the problem that the traditional method does not accurately classify images. The detailed structure is as follows:

1. Using the improved neighborhood averaging method and the wavelet inverse transform respectively, the low frequency subband image and the high frequency subband image are de-noised to improve the image quality.

2. The initial segmentation area of the image is obtained by using the concept of color roughness and the incremental region growth method. Then the initial segmentation region is classified and merged to achieve high precision segmentation.

3. According to the application process of deep learning, we combine small feature extraction, nonlinear mapping and image reconstruction into a deep 
convolution neural network system, and get the final disease image superresolution reconstruction results.

4. Experimental results and analysis to verify the effectiveness of the reconstruction.

5. Discussion.

6. Summarize the content of the full text and look forward to the future.

According to the above method, the problem that the human eye cannot distinguish the pests and diseases of similar crops can be avoided, the accuracy of the identification of pests and diseases is effectively improved, and the economic benefit is improved.

\section{Materials and methods}

\section{Image enhancement of crop disease}

In order to effectively remove impulse noise in crop disease image, wavelet transform can be used to decompose the noisy image into low frequency subband and high frequency subband image, and then process them separately.

In the low frequency subband image denoising, the improved neighborhood averaging method is used to filter the low frequency subband image after the wavelet transform. Assuming that the gray value of pixels of crop disease pixels is $w(i, j)$, then the fuzzy distortion can be reduced as far as possible in the denoising process, so that the gray mean value of the original pixel in the window is as follows:

$$
\overline{w(i, j)}=\left\{\begin{array}{l}
w^{\prime}(i, j)\left|w(i, j)-w^{\prime}(i, j)\right|>T \\
w(i, j) \quad \text { else }
\end{array}\right.
$$

Among them,

$$
w^{\prime}(i, j)=\frac{1}{M N} \sum_{i=1}^{M} \sum_{j=1}^{N} w(i, j)
$$

where $w^{\prime}(i, j)$ representative is centered on the pixel $w(i, j)$, the size is the average value of pixels in a window of $M * N$, and $T$ represents the adaptive threshold. $M$ and $N$ represent the length and width of the window, respectively.

According to the calculation of Equations 1 and 2, the improved neighborhood averaging method is used to filter the low frequency subband image after the wavelet transform, and the denoised image is obtained as follows:

$$
f(x, y)=\frac{1}{M^{*} N} * \frac{\overline{w(i, j)} * T}{w^{\prime}(i, j) * \kappa}
$$

Among them, $f(x, y)$ represents the image of crop disease after the low-frequency subband image denoising, and $\kappa$ represents the image enhancement parameters. 
In the high frequency subband image denoising, considering each subband image in a certain direction, so the horizontal high frequency subband image is filtered by the horizontal window, vertical high frequency subband image is filtered by vertical window, high frequency subband images is filtered by using a shaped window (Giuliani et al., 2016). The local energy method of pixel neighborhood is used to judge the pulse noise point, and the point which is larger than the local energy is regarded as the impulse noise point.

Taking the $3 * 3$ window as an example, the pixel $w(i, j)$ neighborhood of the crop disease image is shown in the following case, as shown in Figure 1.

\begin{tabular}{|l|c|c|}
\hline & $(i-1, j)$ & \\
\hline$(i, j-1)$ & $(i, j)$ & $(i, j+1)$ \\
\hline & $(i+1, j)$ & \\
\hline
\end{tabular}

(a)

\begin{tabular}{|l|l|l|}
\hline$(i-1, j-1)$ & & $(i-1, j+1)$ \\
\hline & $(i, j)$ & \\
\hline$(i+1, j-1)$ & & $(i+1, j+1)$ \\
\hline
\end{tabular}

(b)

Figure 1. Pixel $w(i, j)$ neighborhood of crop disease image

When the image pixel $w(i, j)$ neighborhood of the crop disease image pixel in the case of Figure 1a, the local energy in the neighborhood of the pixel $w(i, j)$ is:

$$
E_{1}(i, j)=\left\{\begin{array}{l}
2\left[w(i, j)-w^{\prime}(i, j)\right]^{2}-\left[w(i-1, j)-w^{\prime}(i, j)\right]\left[w(i+1, j)-w^{\prime}(i, j)\right]- \\
{\left[w(i, j-1)-w^{\prime}(i, j)\right]\left[w(i, j+1)-w^{\prime}(i, j)\right]}
\end{array}\right\} \mid
$$

When the image pixel $w(i, j)$ neighborhood of the crop disease image pixel is in the case of Figure $1 b$, the local energy in the neighborhood of the pixel $w(i, j)$ is:

$$
E_{2}(i, j)=\mid\left\{\begin{array}{l}
2\left[w(i, j)-w^{\prime}(i, j)\right]^{2}-\left[w(i-1, j+1)-w^{\prime}(i, j)\right]\left[w(i+1, j-1)-w^{\prime}(i, j)\right]- \\
{\left[w(i-1, j-1)-w^{\prime}(i, j)\right]\left[w(i+1, j+1)-w^{\prime}(i, j)\right]}
\end{array}\right\}(\operatorname{Eq} .5)
$$

According to Equations 4 and 5, the local energy $E(i, j)$ of the center pixel of the crop disease image center in the neighborhood window is to select the maximum value of $E_{1}(i, j)$ and $E_{2}(i, j)$ as follows: 


$$
E(i, j)=\max \left[E_{1}(i, j), E_{2}(i, j)\right]
$$

It is assumed that the local energy threshold in the neighborhood window is as follows:

$$
T H=\frac{k}{M^{*} N} \sum_{i}^{M} \sum_{j}^{N} E(i, j)
$$

The $k$ in Equation 7 represents the empirical constant. Then it is possible to judge whether a point is a pulse noise point.

$$
E(i, j)>T H
$$

The pulse noise of the satisfying Equation 8 is filtered out by a median filter. An adaptive wavelet threshold is set as follows:

$$
T H_{l}=C_{h} \sigma_{l} \sqrt{\frac{2 \log l+1}{l}} * 2^{\frac{l+1}{2}}
$$

Among them, $l$ represents the decomposition scale, $C_{h}$ represents the constant, $h$ value is $1,2,3$, respectively, which indicates that the horizontal and vertical directions are all high frequency sub-band images, the horizontal direction is the high frequency subband image and the vertical direction is the low frequency subband image, and the horizontal direction is the low frequency subband image and the vertical direction is the high frequency subband image. $\sigma_{l}$ represents the standard deviation of the image noise of crop diseases at various scales, and the wavelet coefficients within each sub window of each scale are as follows:

$$
\sigma_{l}=\left\{\frac{T H_{l}}{M^{*} N} * \sum_{i}^{M} \sum_{j}^{N} w^{\prime}(i, j)-E\left[w_{l}^{\prime}(i, j)\right]\right\}^{\frac{1}{2}}
$$

Among them, $E\left[w_{l}^{\prime}(i, j)\right]$ represents the mean value of the medium and small wave coefficients of each sub window.

Based on the above content, the wavelet coefficients are obtained by using the wavelet soft threshold function to get the enhanced wavelet coefficients.

$$
\eta[w(i, j)]=\operatorname{sgn}[w(i, j)][|w(i, j)|] * \sigma_{l} *\left|T H_{k}\right|
$$

Among them, $\eta$ represents the enhanced wavelet coefficients. The setting of the coefficient can effectively improve the effect of image enhancement. 
According to the enhanced wavelet coefficients obtained by Equation 11, the image $f^{\prime}(x, y)$ of high frequency sub-band crop disease after de-noising is obtained by using the inverse wavelet transform Equation 11.

$$
f^{\prime}(x, y)=E(i, j) * T H_{k} * \frac{\eta[w(i, j)]}{T H}
$$

\section{Image segmentation of crop disease}

According to the image enhancement results of crop disease, the image segmentation algorithm of crop disease is realized by using the image segmentation algorithm of color and image spatial information. The image used in the segmentation is color image, and the whole process is divided into initial segmentation and hierarchical region merging.

The initial segmentation of the image is done by color quantization and incremental regional growth, which will be described in detail below. In order to make the algorithm for the definition of color distance conforms to the human visual characteristics, we choose LUV color space, and the color distance is measured by Euclidean distance (Lal et al., 2016).

For color quantization, it is assumed that a crop disease image contains $M$ windows of $w_{1} * w_{2}$, and each window contains $n$ pixels. $S_{m}$ represents the roughness of the image color, which is used to represent the intensity of the color change in the current window. The color quantization series of the current image is determined by the average color roughness $S_{\text {avg }}$ of the entire image. There are:

$$
\begin{gathered}
S_{m}=\left(\frac{1}{n} \sum_{i=0}^{n-1}\left\|\vec{x}_{i}-\vec{x}_{\text {mean }}^{(m)}\right\|\right)^{\frac{1}{2}} * f^{\prime}(x, y) \\
S_{\text {avg }}=\frac{1}{M} \sum_{i=0}^{M-1} S_{m}
\end{gathered}
$$

where $\overrightarrow{x_{i}}$ represents the color of the $i$ pixel in the $w_{1} * w_{2}$ window, $\vec{x}_{\text {mean }}^{(m)}$ represents the mean color in a window, and $\|\cdot\|$ represents the Euclidean distance. Based on Equation 14, the quantity of the color quantization of a crop disease image is calculated by using Equation 15.

$$
N_{\text {Quan }}=\beta * S_{\text {avg }}+1
$$

Among them, $\beta$ represents the image color quantization threshold, depending on the accuracy of image segmentation, it can be divided into high, medium and low grades, respectively, and is set to 2,1 , and 0.5 , respectively.

After determining the number of color quantization, we use vector quantization to divide the crop disease image pixels into color space, and get the image $\gamma(x, y)$ after color quantization. The formula is as follows: 


$$
\gamma(x, y)=V Q * \sqrt{\frac{N_{\text {Quan }}}{w_{1} * w_{2}}}
$$

Among them, $V Q$ represents vector quantization.

Being divided into the same class of pixels in the color space does not necessarily belong to the same region (Olafsson et al., 2017). These color-classified pixels also need to combine space information to form meaningful segmentation areas. An incremental regional growth algorithm is used to discover the spatial connectivity between pixels. The pixels that conform to the following conditions to form the seed region are defined as:

1. The color is the same after the color is quantified.

2. Four neighborhoods are connected in space.

3. Connected pixels reach a certain area (according to the segmentation precision (that is, high, medium, low), set the original image area of $0.001 \%, 0.01 \%$ and $0.1 \%$ respectively).

The pixels in the image that are not in the seed region will be allocated by a regional growth method to the region that is adjacent to the color and is the closest to the color. For a seed area, if the newly grown pixels meet the following conditions, the algorithm divides the newly growing connected pixels into an independent new seed area. A new region growing method, which can generate new seed regions in growth process is defined as incremental region growing. This growth algorithm is more conducive to some details in the process of growth.

1. The newly grown pixels are connected in the four neighborhoods in space.

2. The average distance between the newly grown pixels is less than the average distance between the pixels and the current region.

3. The newly grown connected pixels reach the requirement of the seed area. (According to the segmentation accuracy (high, middle and low), they are set to $0.001 \%, 0.01 \%$ and $0.1 \%$ of the original image area, respectively).

Through the above calculation and analysis, regional merger is carried out. After the initial segmentation, there are still a lot of similarities between the regions (Katsevich and Katsevich, 2017). The ensemble is combined by a hierarchical region merging algorithm to merge the divided area, and an automatic stop region merging rule is proposed.

For region merging, region distance measurement is an important criterion for region merging. Distance metric determines the result of region merging and the final segmentation result of image. This study considers that the necessary conditions for merging two regions are: two regions are similar in color and adjacent in space, and there is no significant edge on their adjacent areas (Pagnanelli and Borgesneto, 2016). Then the edge, color and adjacency information are fused, and Equation 17 gives the distance definition of the two regions.

$$
D_{i j}^{r}=\left(D_{i j}^{c}\right)^{p} *\left(D_{i j}^{e}\right)^{q} * \Delta_{i j} * \gamma(x, y)
$$


Among them, $p$ and $q$ are used to adjust the color distance and the distance from the edge of the effect size threshold, $\Delta_{i j}$ represents the adjacency relation, that is, the existence of adjacency relation between representatives of $r_{i}$ and $r_{j}$, there is relationship between adjacent assumptions, the $\Delta_{i j}$ value is 1 , whereas the value is $+\infty$, and the region distance is $+\infty . D_{i j}^{c}$ and $D_{i j}^{c}$ represent the color distance and edge distance of two regions, defined as Equations 18 and 19.

$$
\begin{gathered}
D_{i j}^{c}=\frac{\left|r_{i}\right| *\left|r_{j}\right|}{\left|r_{i}\right|+\left|r_{j}\right|} *\left\|\mu_{i}-\mu_{j}\right\| * \sqrt{D_{i j}^{r}} \\
D_{i j}^{e}=\frac{1}{\left|E_{i j}\right|} \sum\left\|x_{a}-x_{b}\right\| * \sqrt{D_{i j}^{c}}
\end{gathered}
$$

Among them, $\left|r_{i}\right|$ and $\left|r_{j}\right|$ represent the number of pixels contained in the $i$ region and the $j$ region, $\mu_{i}$ and $\mu_{j}$ represent the two regional color mean, and the $\left\|\mu_{i}-\mu_{j}\right\|$ represents the Euclidean distance. The product of the molecular part $\left|r_{i}\right|$ and $\left|r_{j}\right|$ in Equation 18 is beneficial to the priority merging of the cell domain, making the segmentation results more consistent with the visual characteristics of the masses. $E_{i j}$ represents the pixels on the edge of two regions, and $\left|E_{i j}\right|$ represents the number of pixels on the edge. $x_{a}$ and $x_{b}$ represent the color values of the $a$ and $b$ points on both sides of the edge, respectively.

For the initial segmentation area, the classification region merging algorithm is used to complete the final segmentation. That is, each time we merge the two regions with the closest distance in the image, form a new area, and the adjacency and distance between the new area and other regions will also be adjusted.

In the process of regional merger, it is a very critical question when to stop the regional merger to get a reasonable combination result, and directly decide the result of image segmentation ( $\mathrm{Wu}$ et al., 2016). The threshold method is used to terminate the region merging, and the merging is affected by the distance between the local regions. The threshold selection also needs to be adjusted according to different images. It is necessary to manually adjust the number of regions according to different requirements, using the method of specifying the number of areas to terminate the merger. Considering the relationship between the information loss in the process of image merging and the number of areas retained in the image, a new automatic stopping region merging criterion is proposed. The detailed process is as follows:

A function that represents the color divergence of the image region is defined to indicate the degree of disunity of the total color in each region of the image. When the area of the image is merged to $K$, the color divergence can be defined as:

$$
J=\frac{\sum_{r=0}^{K} J_{w}^{r}}{J_{t}}=\frac{\sum_{r=0}^{K} \sum_{i=0}^{N_{r}}\left\|x_{i}^{r}-x_{\text {mean }}^{r}\right\|}{\left\|x_{i}^{r}-x_{\text {mean }}^{r}\right\|}
$$


where $J_{w}^{r}$ represents the color divergence within a region, and $J_{t}$ represents the total color divergence in the image. $N_{r}$ represents the number of pixels in the $r$ region, and $x_{i}^{r}$ represents the color value of the $i$ pixel in the $r$ region, and $x_{\text {mean }}^{r}$ represents the color mean of the $r$ region.

Each initial region that defines an image contains only one color, and the color value of all the pixels in each region is equal to the mean color of the region. At this point, the region color of the image is 0 , that is, $J=0$. With the continuous consolidation of the region, that is, the $K$ continues to decrease, and the region color dispersion of the image is increasing in $J$. When all areas in the image are merged into one area, $J$ is 1 . Assuming that $k_{l}$ represents the number of remaining regions in the merging process, the relationship curve between $J$ and $k_{l}$ is shown as shown in Figure 2.

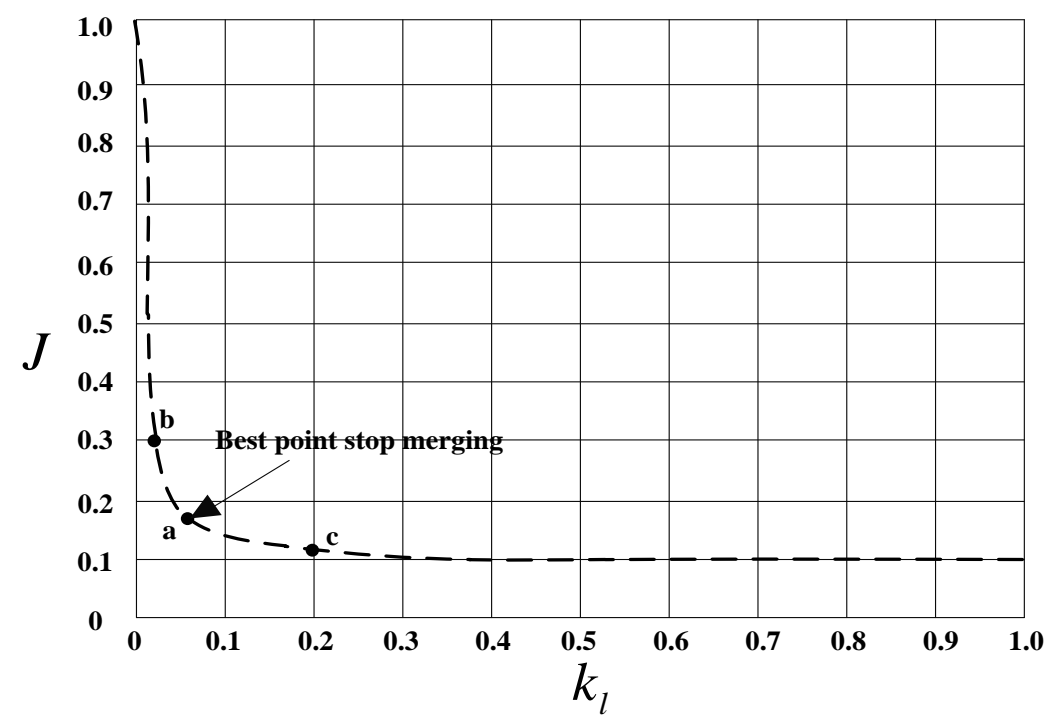

Figure 2. Relation curve between $J$ and $k_{l}$

As shown in Figure 2, from the point of view, it is hoped that the least area can be used to represent the information in an image, that is, the smaller the better the $k_{l}$. From the point of view of the segmentation, the smaller the better the information loss in each region after the segmentation, that is, the smaller the area of the image is, the smaller the $J$, the better. The two values cannot be minimized at the same time, because each area merge will cause the increase of $J$. In order to get the most reasonable segmentation results, the minimum value of $J+k_{l}$ is obtained, that is, the ratio of reserved regions and the color dispersion $J$ of the image area to achieve the best compromise when the $k_{l}$ is terminated. According to the above analysis, the best combination point in Figure 2 is a.

According to the above calculation and analysis, the optimal segmentation results are as follows:

$$
F(x, y)=\min \left(k_{l}+J\right)
$$




\section{Super-resolution reconstruction of crop disease images}

Deep learning is a new field in machine learning research. Its motive is to establish and simulate the human brain's analysis and learning neural network. It simulates the mechanism of human brain to interpret data, such as image, voice and text (Zhou et al., 2016). Based on the image enhancement of 2.1 and the image segmentation of 2.2 , the high resolution reconstruction of the crop disease image is realized by the depth learning method. The concrete process of reconstruction is as follows.

Small feature extraction and representation: it is the first layer of network. The operation will input low resolution image $Y$ to extract small blocks densely, and use high-dimensional feature vectors to represent small blocks. The equivalent is to convolution the image through a set of filters. Assuming that the first layer, the small block feature extraction result is set to $F_{1}$, by using the image segmentation of $2.2, F_{1}$ can be expressed as:

$$
F_{1}(Y)=\max \left(0, W_{1} \partial Y+B\right)
$$

Among them, $W_{1}$ and $B$ represent the bias vector of the filter combination neuron, and $\partial$ represents the convolution operation. Suppose that $d$ represents the number of channels to enter crop disease image. $f_{1}$ represents the spatial size of a single filter, and $n_{1}$ represents the number of filters. The size of $W_{1}$ is $d^{*} f_{1}^{*} n_{1}$.

Through the calculation of Equation 22, $n_{1}$ dimensional feature vector were extracted from each image block, in the second layer nonlinear mapping, each high dimensional vector is mapped to a vector of another dimension $n_{2}$, that is, to transform feature vectors from low resolution space to high resolution space, the output characteristics is $F_{2}(Y)$. Then the mapping results of the second layers can be expressed as:

$$
F_{2}(Y)=\max \left(0, W_{2} \partial F_{1}(Y)+B_{1}\right)
$$

Among them, $W_{2}$ represents convolution kernel, $B_{1}$ represents a $n_{2}$ bias vector, and each output $n_{2}$ dimension vector represents the reconstructed high resolution block.

Using the above calculation, the image block reconstruction layer is reconstructed to form the output image after the mapping. The average can be considered as a predefined filter on a set of feature images, thereby defining the following reconstruction layer to produce high resolution images:

$$
F(Y)=W_{3} \partial F_{2}(Y)+B_{2}
$$

where $W_{3}$ contains $d$ linear filters, and $B_{2}$ represents a bias vector with a dimension of $d$.

Convolution neural network is essentially a mapping from input to output. It can learn a lot of mapping relationships between input and output without any precise mathematical expression between input and output (Salehi et al., 2017). Therefore, as 
long as the convolution network is trained with a known pattern, the network has the ability to map the input and output. The purpose of the convolution neural network is to estimate and optimize the parameters in essence. From the above, the parameters that need to be trained can be expressed as $\theta=\left\{W_{1}, W_{2}, W_{3}, B, B_{1}, B_{2}\right\}$. When the error between the reconstructed image $F(Y ; \theta)$ and the real image $X$ is small, the optimal solution of the parameters can be obtained.

A set of original crop disease image $\left\{X_{i}\right\}$ and its corresponding low resolution image set $\left\{Y_{i}\right\}$ are given, and the loss function is used to indicate the error between them. The mean square error (MSE) is considered to represent the loss function $L(\theta)$. As shown in Equation 25:

$$
L(\theta)=\frac{1}{n} \sum\left\|F(Y ; \theta)-X_{i}\right\|^{2}
$$

According to the calculation by Equation 25, we can see that the error between original crop disease image $\left\{X_{i}\right\}$ and its corresponding low resolution image set $\left\{Y_{i}\right\}$ is the smallest, that is, when the loss function is minimum $\min L(\theta)$, the image reconstruction accuracy of crop disease is the highest. The result of the final image super-resolution reconstruction using the minimum loss function is as follows:

$$
\operatorname{Best}(X, Y)=\min L(\theta) *\left(\left\{X_{i}\right\} \Leftrightarrow\left\{Y_{i}\right\}\right) * \varpi * \vartheta
$$

Among them, $\varpi$ represents the threshold of super resolution reconstruction for crop disease image, which is controlled between 0.4-0.5. After image reconstruction, integrity is kept the best and the reconstruction result is the best. $\vartheta$ represents the speed factor of the image reconstruction convergence speed, which can improve the speed of image reconstruction efficiently.

\section{Results}

In order to verify the effect of reconstruction, the experiment was completed by the contrast of different image reconstruction methods. Taking into account the fairness of comparison, the experiment used a data set of a domestic agricultural production base on crop disease image as a training sample, and the experimental platform was built on Matlab7.0. After training the samples, the experimental objects were tested with the trained parameters, this result is obtained from a real agricultural planting shed and Figure 3 is the test sample.

According to the experimental platform and the experimental data, the experiment is carried out under the following experimental indexes.

1. Image enhancement effect of crop disease, that is, image clarity.

2. The accuracy of image segmentation.

3 . The integrity of the reconstructed image.

4. The convergence rate of image reconstruction. 


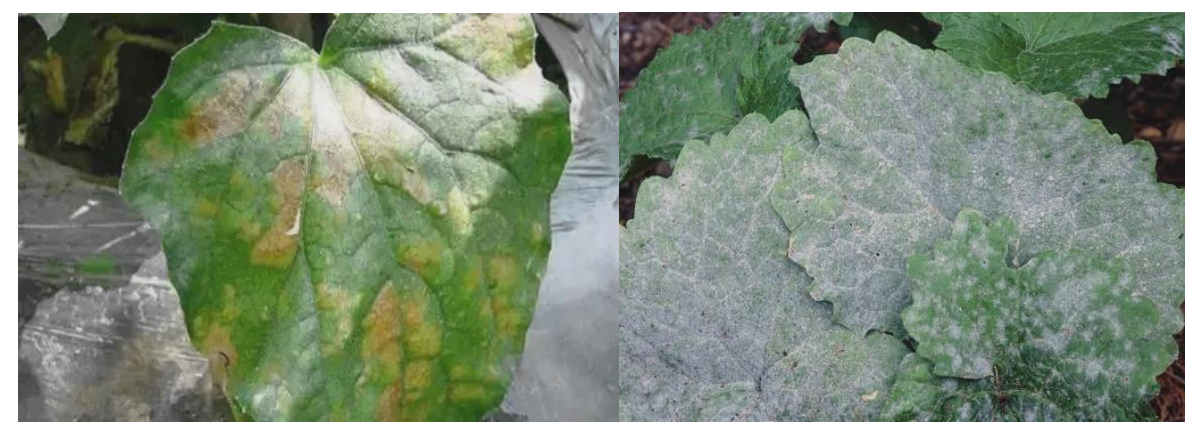

Figure 3. Test examples

The experimental results are as follows:

Figure 4 showed that image reconstruction based on support vector machine has low clarity and low color division, and texture area is poor. The image reconstruction method based on depth learning is very effective, the texture is clear and the color is distinct. The overall definition of the image is better than the image reconstruction method based on support vector machine.

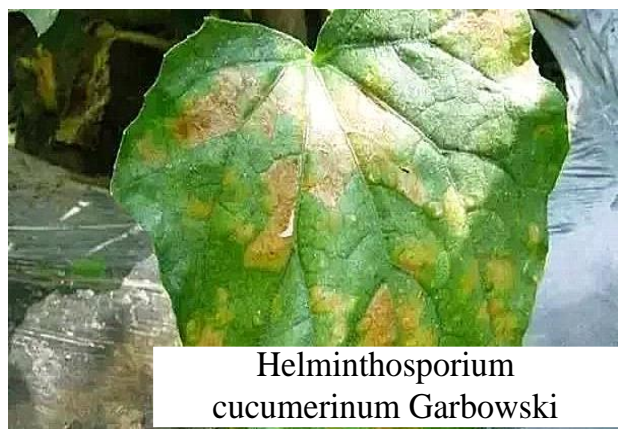

(a) Image enhancement effect based on support vector machine image reconstruction

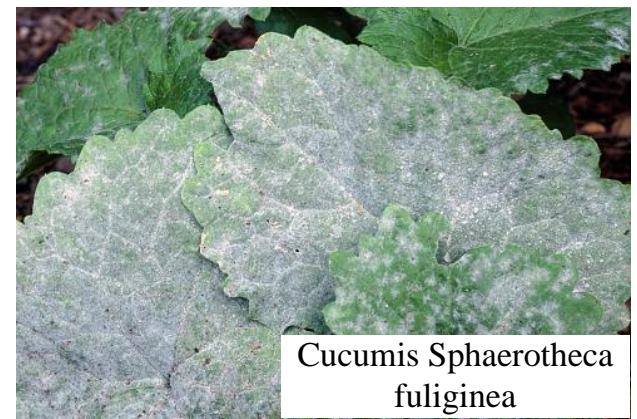

(b) Image enhancement effect based on deep learning image reconstruction

Figure 4. Enhancement of image reconstruction with different image reconstruction methods

According to the analysis of Figure 5, the image segmentation accuracy of image reconstruction based on edge gradient information is gradually low with the increase of image number. The maximum segmentation precision is $56 \%$ and the lowest is $33 \%$, which indicates that the reliability of the method is low. The image segmentation accuracy based on wavelet denoising and neural network image reconstruction has a downward trend, the maximum segmentation precision is $63 \%$, and the lowest is $50 \%$. 
The image segmentation precision curve of the image reconstruction method based on depth learning is increasing gradually, the maximum segmentation precision is $96 \%$ and the minimum segmentation precision is $75 \%$, which is more reliable compared with image reconstruction method based on the edge gradient information.

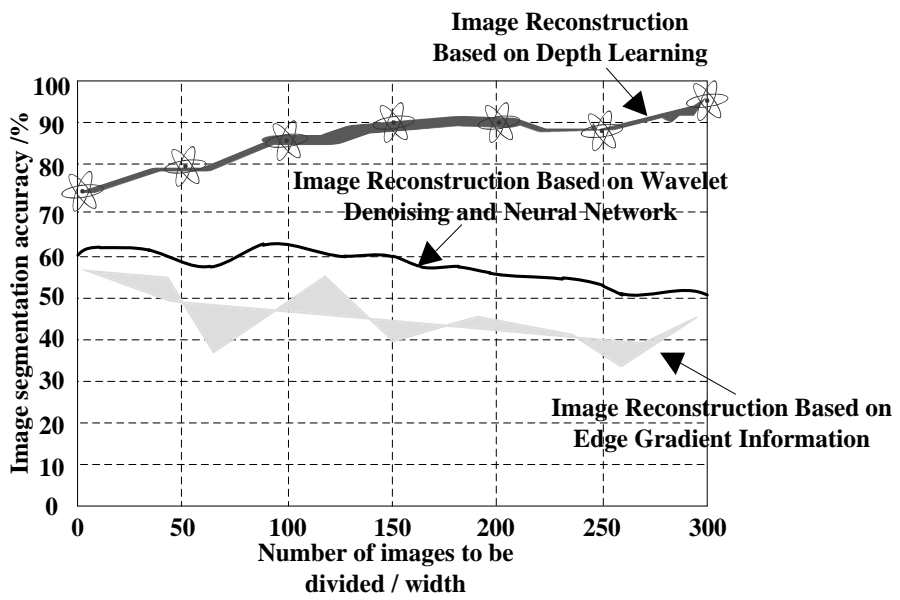

Figure 5. Image segmentation precision comparison of different image reconstruction methods

From the contrast result of Figure 6, we can see that the integrity coefficient of image reconstruction based on compressed sensing is low, the highest is 0.48 . This data indicates that the integrity of image reconstruction is not guaranteed. The integrity coefficient of the reconstruction method based on depth learning is 0.98 and the lowest is 0.7. According to the data comparison, it is obvious that the image reconstruction based on depth learning can be practicable and useful for reference.

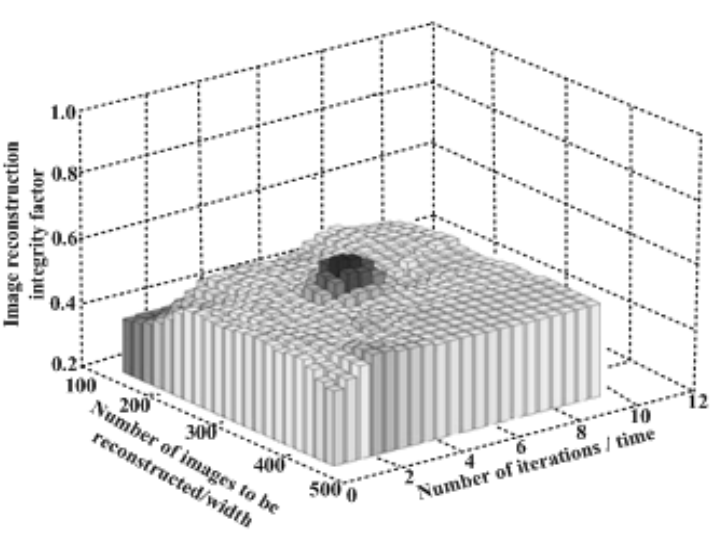

(a) Integrity of image reconstruction based on compressed sensing

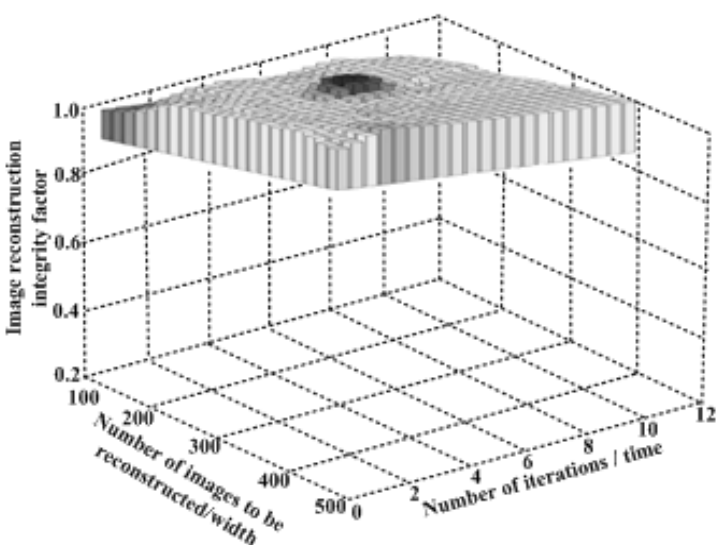

(b) Integrity of image reconstruction based on deep learning

Figure 6. Reconstruction integrity comparison of different image reconstruction methods

In Figure 7, the time-consuming curve of the image reconstruction convergence based on Tabu search showed an unstable state, which is fluctuate up and down, and the average convergence time is about $0.5 \mathrm{~s}$. The time-consuming curve stability of the image reconstruction based on depth learning is better, the maximum time of 
convergence is $0.35 \mathrm{~s}$, and the time of average convergence is $0.3 \mathrm{~s}$. Compared with the two image reconstruction methods, the time dependent data is convergent, and the image reconstruction method based on depth learning occupies a significant advantage and is reliable.

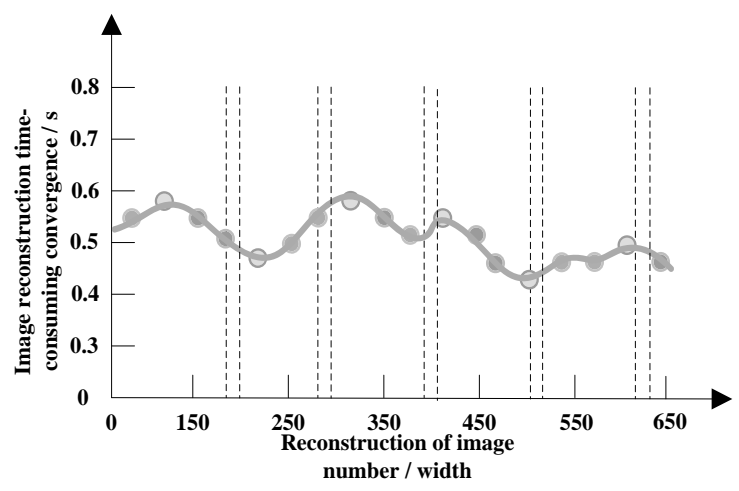

(a) Time consuming of image reconstruction based on Tabu Search

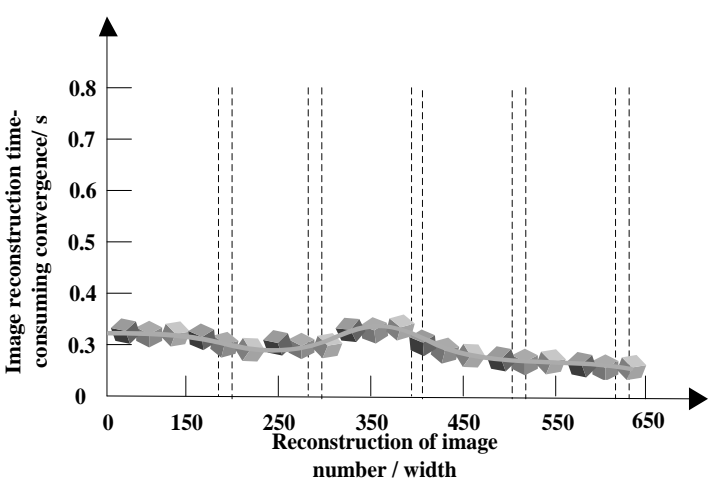

(b) Time consuming of image reconstruction based on deep learning

Figure 7. Time consuming comparison of reconstruction of different image reconstruction methods

The identification of crop diseases is accurately compared, and the results are as follows:

Analysis of Figure 8 shows that the accuracy of the identification of disease and insect information in three different methods is different. The accuracy of this method is significantly higher than the other two methods, and has a high promotion value.

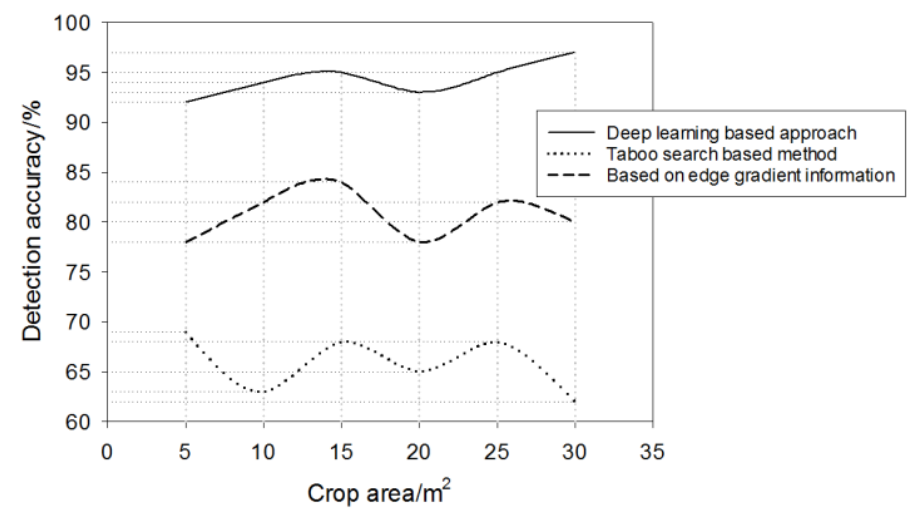

Figure 8. Comparison of information recognition accuracy under different methods

\section{Discussion}

In the discussion part, the $\varpi$ threshold of super resolution reconstruction of crop disease image is used to observe and verify the influence of the value range on the super resolution reconstruction of crop image.

In order to make the results of the discussion more comprehensive, the $\varpi$ values of $0.3,0.4$ and 0.45 are considered as the scope of discussion, respectively. The discussion environment is selected as follows: Intel (R) Core (TM) i5-3210M CPU@2.50 GHz; 
6.00 GB memory; Win7x64; Matlab; Caffe. And the Matlab interface of Caffe is used to achieve image super resolution reconstruction.

The graphs in Figure 9 represent the effects on image reconstruction when the $\varpi$ values are $0.3,0.4$, and 0.45 .

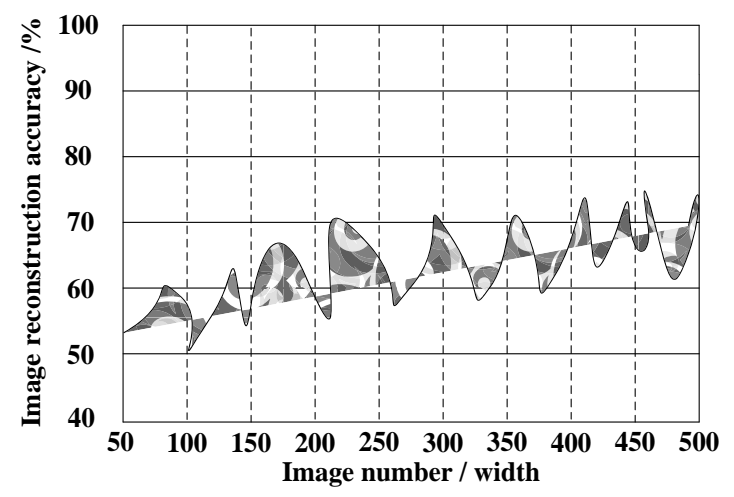

(a) Image reconstruction accuracy when $\varpi$ is 0.3

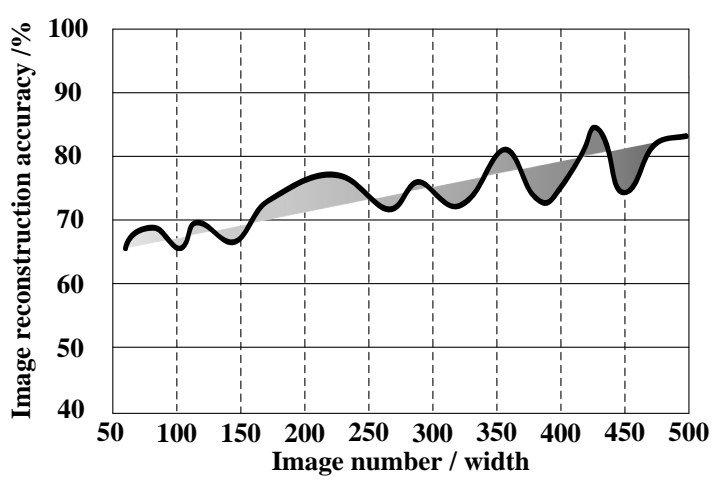

(b) Image reconstruction accuracy when $\varpi$ is 0.4

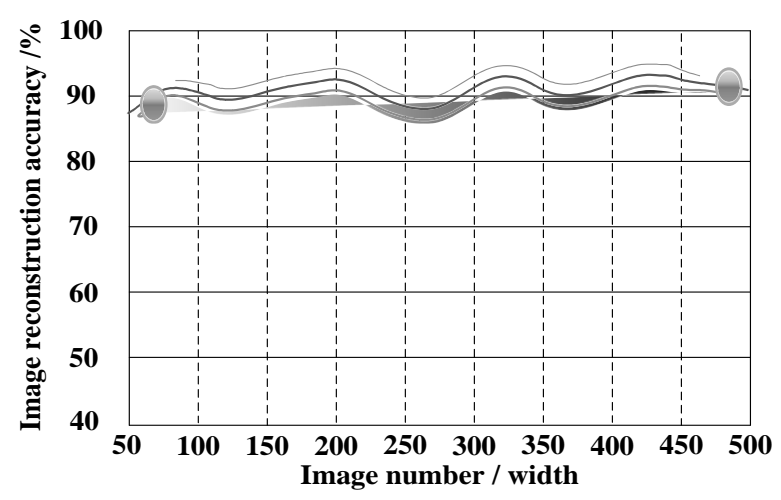

(c) Image reconstruction accuracy when $\varpi$ is 0.45

Figure 9. The impact of different values of $\varpi$ on image reconstruction results

According to the results of Figure 9, we can see that when $\varpi$ is 0.3 , the accuracy of image reconstruction is $73 \%$, and the accuracy of reconstruction accuracy is not good, but it shows a rising trend. When the $\varpi$ is 0.4 , the accuracy of image reconstruction is obviously superior to that of when $\varpi$ is 0.3 , the precision of the reconstruction is $83 \%$, and the whole fluctuation of the precision curve is stable. When the $\varpi$ is 0.45 , the accuracy of the image reconstruction is the highest and the transformation of the precision curve is the smallest. When the reconstructed image is 425, the reconstruction precision is $95 \%$. The above data show that when $\varpi$ is 0.45 , the image reconstruction results are most significant.

\section{Conclusions}

At present, there are many problems in the algorithms and methods of image reconstruction. In order to solve these problems, an image super-resolution reconstruction based on deep learning is proposed. Image reconstruction is completed by three links of image enhancement, image segmentation and image reconstruction. In the process of image reconstruction, the speed of reconstruction and the accuracy of 
reconstruction are the difficult problems. In order to solve such problems, the threshold of image super-resolution reconstruction and the speed factor of image reconstruction convergence are set up respectively. Two parameters are used to control the accuracy and speed of the reconstruction. The effectiveness of the proposed image reconstruction method is verified by experiments and discussions. It is also proved that the threshold of reconstruction and the speed of convergence play a very important role in the whole process of image reconstruction, which effectively solves the problems of image reconstruction.

In the future, the efficiency of image reconstruction will be further studied while compressing reconstruction costs.

\section{REFERENCES}

[1] Burgess, A. J., Retkute, R., Pound, M. P., Mayes, S., Murchie, E. H. (2017): Image-based $3 \mathrm{D}$ canopy reconstruction to determine potential productivity in complex multi-species crop systems. - Annals of Botany 119: 517-532.

[2] Fu, H., Liu, X. (2017): A Study on the impact of environmental education on individuals' behaviors concerning recycled water reuse. - Eurasia Journal of Mathematics Science and Technology Education 13(10): 6715-6724.

[3] Gao, W., Baig, A. Q., Ali, H., Sajjad, W., Farahani, M. R. (2017): Margin based ontology sparse vector learning algorithm and applied in biology science. - Saudi Journal of Biological Sciences 24(1): 132-138.

[4] Giuliani, M., Vissa, A., Driouchi, A., et al. (2016): Effect of data pre-processing on super-resolution reconstruction and pattern recognition. - Biophysical Journal 110(3): 331a.

[5] Guo, B. H., Cen, Z. S. (2016): Super resolution image reconstruction based on wavelet denoising and neural network. - Laser Journal 37: 61-64.

[6] Katsevich, A., Katsevich, A. (2017): A local approach to resolution analysis of image reconstruction in tomography. - Siam Journal on Applied Mathematics 77: 1706-1732.

[7] Kniat, A. (2017): Visualization of a lifeboat motion during lowering along ship's side. Polish Maritime Research 24(4): 42-46.

[8] Lal, A., Shan, C., Xi, P. (2016): Structured illumination microscopy image reconstruction algorithm. - IEEE Journal of Selected Topics in Quantum Electronics 22: 50-63.

[9] Li, C. (2016): Super resolution image reconstruction based on support vector machine. Laser Journal 37: 138-141.

[10] Mao, X. D., Shi, Z. (2016): Method about reconstruction of SEM image based on gradient. - Computer Science 43: 297-301.

[11] Mo, S., Li, Z., Gou, K., Qin, L., Shen, B. (2018): Quantifying the effects of climate variability and direct human activities on the change in mean annual runoff for the Bahe River (Northwest China). - Journal of Coastal Research 34(1): 81-89.

[12] Nongqwenga, N., Modi, A. T. (2017): Phosphorus and potassium quantity/intensity properties of selected South African soils (Kwazulu-Natal) and their correlation with selected soil parameters. - Applied Ecology and Environmental Research 15(3): 1-14.

[13] Olafsson, V. T., Noll, D. C., Fessler, J. A. (2017): Fast spatial resolution analysis of quadratic penalized least-squares image reconstruction with separate real and imaginary roughness penalty: application to fMRI. - IEEE Transactions on Medical Imaging. DOI: 10.1109/TMI.2017.2768825.

[14] Pagnanelli, R., Borgesneto, S. (2016): Technical aspects of resolution recovery reconstruction. - Journal of Nuclear Cardiology 23: 149-152. 
[15] Roy, S., Handique, G., Bora, F. R., Rahman, A. (2018): Evaluation of certain nonconventional plant based oils against red spider mite of tea. - Journal of Environmental Biology 39(1): 1-4.

[16] Salehi, B., Daneshfar, B., Davidson, A. M. (2017): Accurate crop-type classification using multi-temporal optical and multi-polarization SAR data in an object-based image analysis framework. - International Journal of Remote Sensing 38: 4130-4155.

[17] Sanchez Camacho, E. A., Martinez Morales, M. (2017): Estimation of the volume of underground water for a coastal wetland. - Revista Internacional De Contaminacion Ambiental 33(SI): 65-76.

[18] Song, J. H. (2016): Image reconstruction of SRM cross-section based on compressed sensing. - Computer Simulation 33: 132-137.

[19] Wei, D. (2017): Image super-resolution reconstruction using the high-order derivative interpolation associated with fractional filter functions. - Iet Signal Processing 10: 10521061.

[20] Wu, G. H., Zhang, H. D., Li, X. K., Dai, J. Y. (2016): FR-CoSaMP image reconstruction algorithm based on improved measurement. - Computer Engineering and Design 37: 1555-1559.

[21] Yu, J. P., Chen, D. Y., Wang, L. L. (2016): A Novel image reconstruction algorithm based on improved taboo search for electrical capacitance tomography. - Journal of Harbin University of Science and Technology 21: 51-56.

[22] Zhou, L. Y., Su, C. X., Cao, Y. F. (2016): Image super-resolution via sparse representation. - Computer Engineering and Design 37: 3290-3294. 